\title{
Calculating the density of electronic charge for hydrogen atom and ions like atom
}

\author{
Ban H. Adel* Khalil A. Al-Bayati* Nessrin K. Abd-AL Ameer* \\ Received 3, January, 2011 \\ Accepted 7, June, 2011
}

\begin{abstract}
:
The radial wave function $\mathrm{R}(\mathrm{r})$ and the radial distribution function $\mathrm{P}(\mathrm{r})$ as a function of (r), for the Hydrogen atom was calculated for several atomic state $(1 \mathrm{~s}, 2 \mathrm{~s}, 2 \mathrm{p}, 3 \mathrm{~s}, 3 \mathrm{p}, 3 \mathrm{~d}) \quad$ The results were compared with Hydrogen like atom $\left(\mathrm{He}^{+}, \mathrm{Li}^{+2}, \mathrm{Be}^{+3}\right)$.
\end{abstract}

Key words : Hydrogen atom, radial wave function, radial distribution function

\section{Introduction :}

The radial distribution function for $\mathrm{He}$ atom and like ions has been calculated by Al-bayati \&Ban [1]. The electronic wave function, describes the distribution of the electron positions in terms of the distance of the electron from the nucleus, $\mathbf{r}$, and the orientation of the electron relative to the nucleus. We can separate the wave function into an orientationally dependent part, known as the angular wave function, and an orientationally independent part, which is known as the Radial Wave Function [2].

$\varphi_{n l m_{s}}(r, \theta, \varnothing)=R_{n l}(r) Y_{l m_{l}}(\theta, \varnothing)$

The Radial Wave Function, $\mathrm{R}_{\mathrm{nl}}(\mathrm{r})$, depends only on the distance of the electron from the nucleus, and is characterized by the values of the principal and orbital angular quantum numbers, whereas the angular wave function, Y, depends on the angles of the electron from the nucleus, and is characterized by the values of the orbital angular and magnetic quantum numbers. The $s$ orbital consist only of a radial part to the wave function.
The angular wave functions for other types of orbital are complicated mathematical expressions, so generally only the shapes of the orbital, are important. Another important function we need to consider is the Radial Distribution Function, $\mathrm{P}_{\mathrm{nl}}(\mathrm{r})$. This is defined as the probability that an electron in the orbital with quantum numbers $\mathrm{n}$ and 1 will be found at a distance $r$ from the nucleus. It is related to the radial wave function by the following relationship[3]:

$P_{n l}(r)=4 \pi^{2} R_{n l}(r) ;$ normalized $\ldots \int_{0}^{\infty} P_{n l}(r) d r=1$

The same procedure, of separation of variables, is possible for other atomic species e.g. $\mathrm{He}^{+}, \mathrm{Li}^{+2}$ and $\mathrm{Be}^{+3}$, considered to the extent that there is one extra-nuclear electron.

\section{Radial Distribution function for the Hydrogen atom:}

Often it is more meaningful physically to make plots of the radial distribution function $\mathrm{P}(\mathrm{r})$, of an atomic orbital, since this display emphasizes the spatial reality of the probability 
distribution of the electron density as shell structure about the nucleus.To establish the radial distribution function we need to calculate the probability of an electron, in particular orbital, exhibiting coordinates on a thin shell of width.

The calculations and results:

Table (1) - The radial components of the hydrogenic atomic orbitals[4]

\begin{tabular}{|c|c|c|c|}
\hline $\begin{array}{c}\text { orbit } \\
\text { al }\end{array}$ & $\mathbf{N}_{\mathbf{R}}$ & & \\
\hline $1 \mathrm{~s}$ & $2\left(\frac{Z}{a}\right)^{3}$ & & \\
\hline $2 \mathrm{~s}$ & $\left(\frac{1}{2(2)^{1 / 2}}\right)$ & $\left(\frac{z}{a}\right)^{3}$ & $\begin{array}{l}(2 \\
-\rho 1\end{array}$ \\
\hline $2 p$ & $\left(\frac{1}{2(6)^{1 / 2}}\right)$ & $\left(\frac{Z}{a}\right)^{3}$ & \\
\hline $3 \mathrm{~s}$ & $\left(\frac{1}{9(3)^{1 / 2}}\right)$ & $\left(\frac{Z}{a}\right)$ & \\
\hline $3 p$ & $\left(\frac{1}{9(6)^{1 / 2}}\right)$ & $\left(\frac{z}{a}\right)^{3}$ & $\begin{array}{l}(4 \rho 1 \\
-(\beta\end{array}$ \\
\hline $\mathbf{r}$ & RH(r) & PH(r & \\
\hline 0 & 2 & 0 & \\
\hline 0.1 & 1.809674836 & 0.03274 & 4923 \\
\hline 0.2 & 1.637461506 & 0.10725 & 1207 \\
\hline 0.3 & 1.481636441 & 0.197571 & 2189 \\
\hline 0.4 & 1.340640092 & 0.28757 & 0537 \\
\hline 0.5 & 1.213061319 & 0.36787 & 9441 \\
\hline 0.6 & 1.097623272 & 0.43371 & 9665 \\
\hline 0.7 & 0.993170608 & 0.48333 & $\overline{0049}$ \\
\hline 0.8 & 0.898657928 & 0.51685 & 5086 \\
\hline 0.9 & 0.813139319 & 0.53556 & 8398 \\
\hline 1 & 0.735758882 & 0.54134 & 1133 \\
\hline 1.1 & 0.665742167 & 0.53628 & 7286 \\
\hline 1.2 & 0.602388424 & 0.52253 & 5411 \\
\hline 1.3 & 0.545063586 & 0.50208 & 9389 \\
\hline 1.4 & 0.493193928 & 0.47675 & 0891 \\
\hline 1.5 & 0.44626032 & 0.44808 & 3615 \\
\hline 1.6 & 0.403793036 & 0.41740 & 4969 \\
\hline 1.7 & 0.365367048 & 0.38579 & 5001 \\
\hline 1.8 & 0.330597776 & 0.35411 & 5443 \\
\hline 1.9 & 0.299137238 & 0.32303 & 3946 \\
\hline 2 & 0.270670566 & 0.29305 & 0222 \\
\hline 2.1 & 0.244912857 & 0.26452 & 1975 \\
\hline 2.2 & 0.221606317 & 0.23768 & 9301 \\
\hline 2.3 & 0.200517687 & 0.21269 & 6844 \\
\hline 2.4 & 0.181435907 & 0.18961. & 3372 \\
\hline 2.5 & 0.164169997 & 0.16844 & 8675 \\
\hline 2.6 & 0.148547156 & 0.14916 & 7902 \\
\hline 2.7 & 0.134411025 & 0.1317 & 035 \\
\hline 2.8 & 0.121620125 & 0.11596 & 5006 \\
\hline 2.9 & 0.11004644 & 0.10184 & 6942 \\
\hline 3 & 0.099574137 & 0.08923 & 5078 \\
\hline
\end{tabular}

\begin{tabular}{|l|l|}
\hline 3d & $\left(\frac{1}{9(30)^{1 / 2}}\right)\left(\frac{Z}{a}\right)(\rho r)^{2} e^{-\rho r / 2}$ \\
\hline
\end{tabular}

The radial component of the hydrogenic atomic orbitals with distinct normalization constants for the radial functions[5]. The parameter $\rho=(2 Z / n * a)$ extents the application of the function in the table (1) entries for non-hydrogen one-electron atomic species.

Remark : the maximum $P(r)$ for $H$ atom accurse at $r=1$ Bohr unit

\begin{tabular}{|c||c|c|c|c|}
\hline Atom & $\mathbf{H}$ & $\mathbf{H e}$ & $\mathbf{L i}$ & $\mathbf{B e}$ \\
\hline $\mathbf{Z}$ & 1 & 2 & 3 & 4 \\
\hline $\boldsymbol{\rho}$ & 2 & 4 & 6 & 8 \\
\hline
\end{tabular}
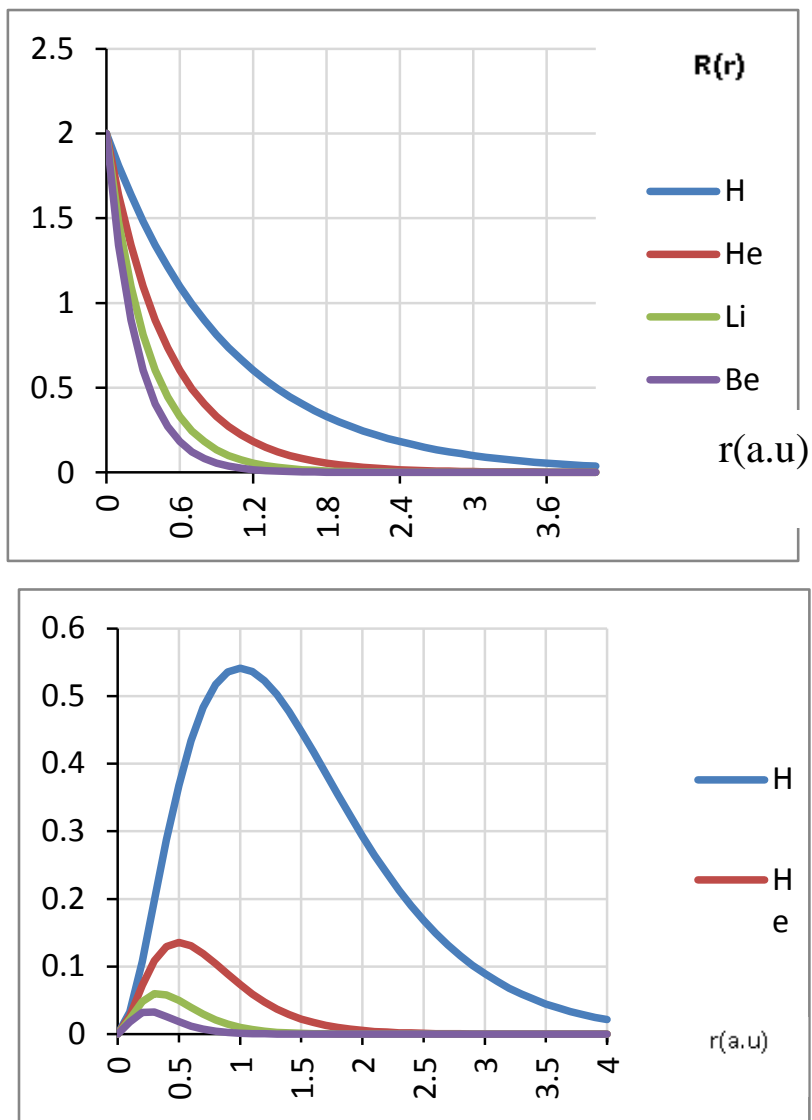


\begin{tabular}{|l|c|c|}
3.1 & 0.090098405 & 0.078011314 \\
\hline 3.2 & 0.081524408 & 0.068057386 \\
\hline 3.3 & 0.073766335 & 0.059257632 \\
\hline 3.4 & 0.06674654 & 0.051500963 \\
\hline 3.5 & 0.060394767 & 0.044682216 \\
\hline
\end{tabular}

Fig. (1) The normalized radial wave function $R(r)$ and the radial $\mathrm{r}$ distribution function $\mathrm{P}(\mathrm{r})$ for the $1 \mathrm{~s}$ atomic orbital in the hydrogen atom and hydrogen like ions.

\begin{tabular}{|c|c|c|}
\hline 0 & 0.7071 & \\
\hline 0.2 & 0.575829484 & 0.013263184 \\
\hline 0.4 & 0.463139612 & 0.034319728 \\
\hline 0.6 & 0.366682795 & 0.048404258 \\
\hline 0.8 & 0.284389983 & 0.051761704 \\
\hline
\end{tabular}

\begin{tabular}{|c||c|c|c|c|}
\hline Atom & H & He & Li & Be \\
\hline$Z$ & 1 & 2 & 3 & 4 \\
\hline$\rho$ & 1 & 2 & 3 & 4 \\
\hline
\end{tabular}

\begin{tabular}{|l|l|l|}
\hline 0.8 & 0.284389983 & 0.051761704 \\
\hline 1 & 0.214438915 & 0.045984048 \\
\hline & & 0.034696908 \\
\hline
\end{tabular}

\begin{tabular}{|l|l|l|}
\hline 1.2 & 0.155225883 & 0.034696908 \\
\hline 1.4 & 0.10534064 & 0.021749435 \\
\hline 1.6 & 0.063544102 & 0.010336903 \\
\hline
\end{tabular}

\begin{tabular}{|l|l|l|}
\hline 1.6 & 0.063544102 & 0.010336903 \\
\hline 1.8 & 0.028748541 & 0.002677791 \\
\hline & & 0 \\
\hline
\end{tabular}

\begin{tabular}{|l|l|l|}
\hline 2 & 0 & 0 \\
\hline 2.2 & -0.02353731 & 0.002681385 \\
\hline 2.4 & -0.04259489 & 0.010450508 \\
\hline
\end{tabular}

\begin{tabular}{|l|l|l|}
\hline 2.4 & -0.04259489 & 0.010450508 \\
\hline 2.6 & -0.05781217 & 0.022593589 \\
\hline & & 0.03813934 \\
\hline
\end{tabular}

\begin{tabular}{|l|l|l|}
\hline 2.8 & -0.06974749 & 0.03813934 \\
\hline 3 & -0.07888767 & 0.056009378 \\
\hline
\end{tabular}

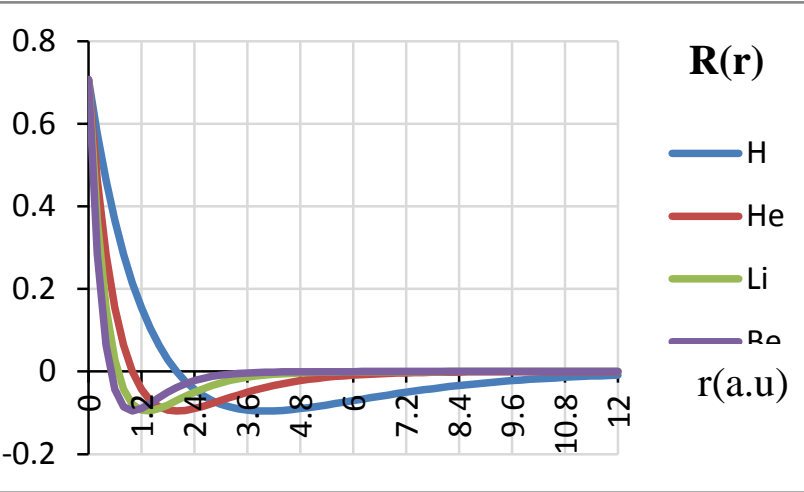

\begin{tabular}{|l|l|l|}
\hline 3.2 & -0.08565662 & 0.075131453 \\
\hline 3.4 & -0.09042286 & 0.094517962 \\
\hline 3.6 & -0.09350628 & 0.113314768 \\
\hline
\end{tabular}

\begin{tabular}{|l|l|l|}
\hline 3.6 & -0.09350628 & 0.113314768 \\
\hline 4.8 & -0.09518397 & 0.130826239 \\
\hline 4.2 & -0.09569558 & 0.146522301 \\
\hline & -0.09524783 & 0.160032725 \\
\hline
\end{tabular}

\begin{tabular}{|l|l|l|}
\hline 4.4 & -0.0940187 & 0.171133014 \\
\hline 4.6 & -0.09216094 & 0.179725386 \\
\hline 4.8 & -0.08980533 & 0.185817541 \\
\hline
\end{tabular}

\begin{tabular}{|l|l|l|}
\hline 4.8 & -0.08980533 & 0.185817541 \\
\hline 5 & -0.08706345 & 0.189501125 \\
\hline 5.2 & -0.08403016 & 0.190931252 \\
\hline 5.4 & -0.08078573 & 0.190307908 \\
\hline 5.6 & -0.07739783 & 0.187859707 \\
\hline 5.8 & -0.07392315 & 0.183830204 \\
\hline 6 & -0.07040887 & 0.178466734 \\
\hline 6.2 & -0.06689401 & 0.172011647 \\
\hline 6.4 & -0.0634105 & 0.164695715 \\
\hline 6.6 & -0.0599842 & 0.15673343 \\
\hline 6.8 & -0.05663577 & 0.148319928 \\
\hline 7 & -0.05338142 & 0.139629248 \\
\hline
\end{tabular}

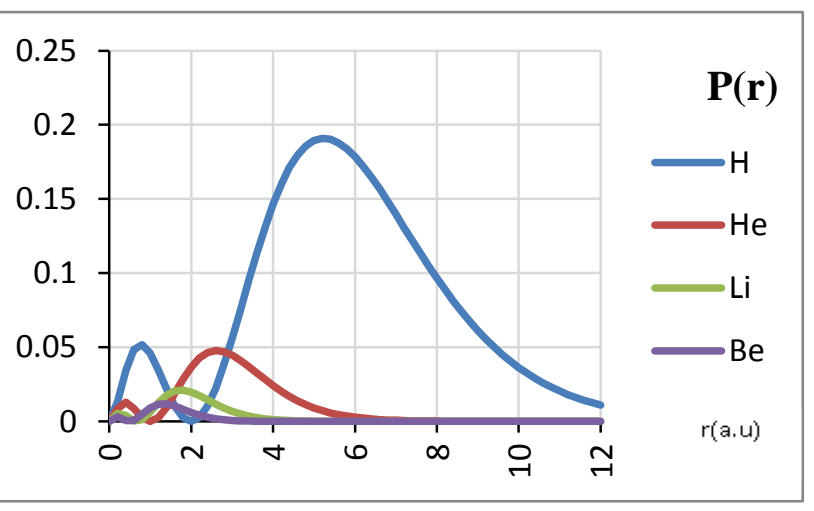

Fig. (2) The normalized radial wave function $R(r)$ and the radial distribution function $P(r)$ for the 2 s atomic orbital in the hydrogen atom and hydrogen like ions. 


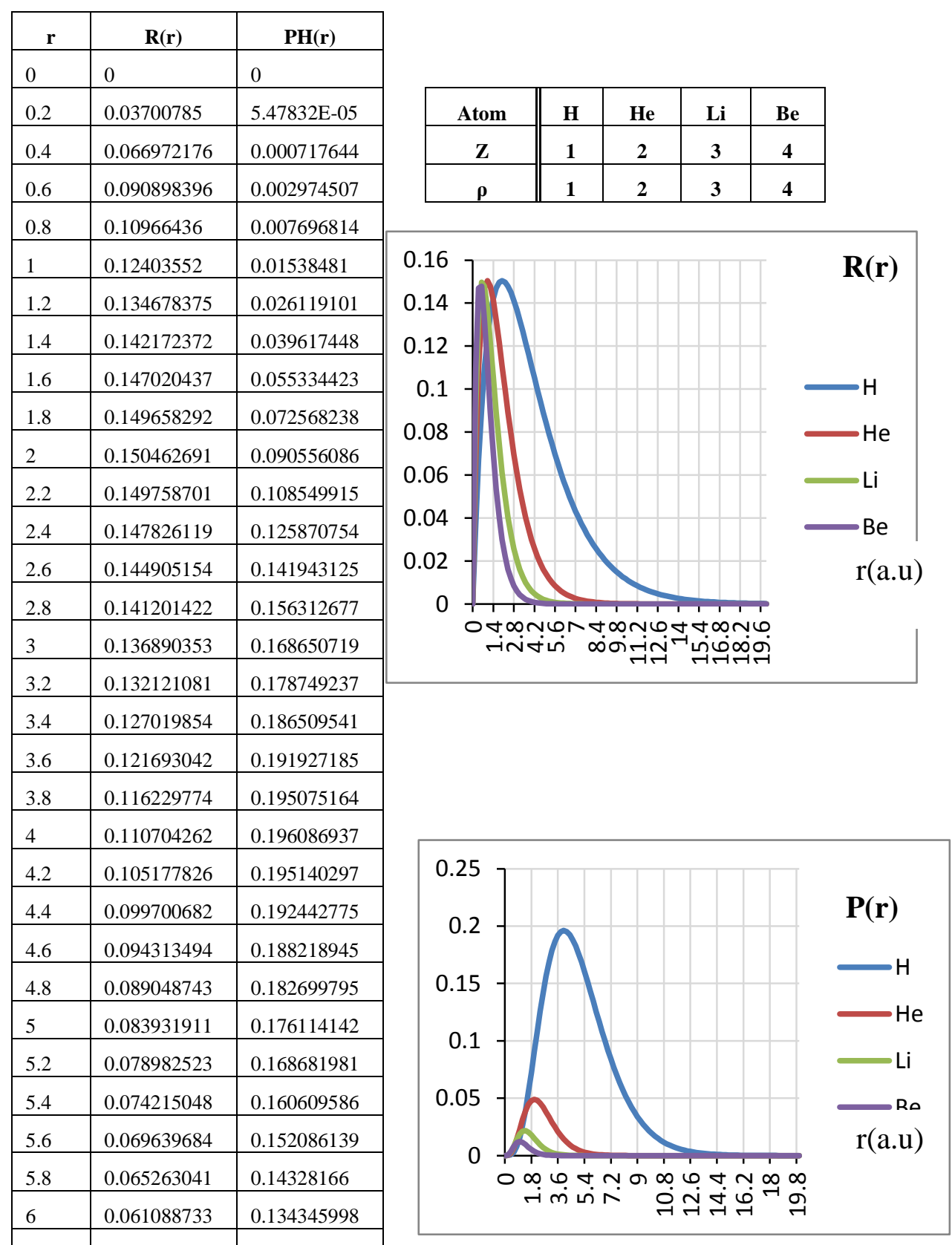

\begin{tabular}{|l|l|l|}
\hline 6.2 & 0.057117884 & 0.125408679 \\
\hline 6.4 & 0.053349573 & 0.116579406 \\
\hline 6.6 & 0.049781211 & 0.10794904 \\
\hline 6.8 & 0.046408869 & 0.099590932 \\
\hline 7 & 0.043227554 & 0.091562451 \\
\hline
\end{tabular}

Fig. (3) The normalized radial wave function $R(r)$ and the radial distribution function $P(r)$ for the $2 p$ atomic orbital in the hydrogen atom and hydrogen like ions. 


\begin{tabular}{|l|l|l|}
\hline \multicolumn{1}{|c|}{$\mathbf{r}$} & \multicolumn{1}{|c|}{$\mathbf{R}(\mathbf{r})$} & \multicolumn{1}{|c|}{$\mathbf{P H}(\mathbf{r})$} \\
\hline 0 & 0.3852 & 0 \\
\hline 0.2 & 0.313377377 & 0.003928215 \\
\hline 0.4 & 0.251214417 & 0.010097389 \\
\hline 0.6 & 0.197635054 & 0.014061461 \\
\hline 0.8 & 0.151670151 & 0.014722454 \\
\hline 1 & 0.112447647 & 0.012644473 \\
\hline 1.2 & 0.079183566 & 0.009028854 \\
\hline 1.4 & 0.051173814 & 0.005132768 \\
\hline 1.6 & 0.027786694 & 0.001976577 \\
\hline 1.8 & 0.00845609 & 0.000231678 \\
\hline 2 & -0.00732475 & 0.000214608 \\
\hline 2.2 & -0.02000888 & 0.001937719 \\
\hline 2.4 & -0.0300008 & 0.005184275 \\
\hline 2.6 & -0.03766115 & 0.00958813 \\
\hline 2.8 & -0.04331101 & 0.014706613 \\
\hline 3 & -0.04723572 & 0.020080919 \\
\hline 3.2 & -0.04968846 & 0.025281982 \\
\hline 3.4 & -0.05089345 & 0.029942052 \\
\hline 3.6 & -0.0510488 & 0.033773507 \\
\hline 3.8 & -0.05032925 & 0.036577009 \\
\hline 4 & -0.04888848 & 0.03824134 \\
\hline 4.2 & -0.04686131 & 0.038737136 \\
\hline 4.4 & -0.04436568 & 0.038106555 \\
\hline 4.6 & -0.04150441 & 0.036450548 \\
\hline 4.8 & -0.0383668 & 0.03391514 \\
\hline 5 & -0.03503013 & 0.030677748 \\
\hline 5.2 & -0.03156093 & 0.02693433 \\
\hline 5.4 & -0.02801618 & 0.022887866 \\
\hline 5.6 & -0.02444439 & 0.018738488 \\
\hline 5.8 & -0.02088657 & 0.014675403 \\
\hline 6 & -0.01737705 & 0.010870628 \\
\hline 6.2 & -0.01394434 & 0.007474448 \\
\hline 6.4 & -0.01061176 & 0.00461248 \\
\hline 6.6 & -0.00739811 & 0.002384124 \\
\hline 6.8 & -0.00431822 & 0.000862237 \\
\hline 7 & -0.00138347 & $9.3785 \mathrm{E}-05$ \\
\hline & $\mathbf{g}$. & $\mathbf{1}$ \\
\hline
\end{tabular}

\begin{tabular}{|c||c|c|c|c|}
\hline Atom & H & He & Li & Be \\
\hline$Z$ & 1 & 2 & 3 & 4 \\
\hline$\rho$ & 0.6667 & $\mathbf{1 . 3 3 3 3}$ & 2 & 2.6667 \\
\hline
\end{tabular}
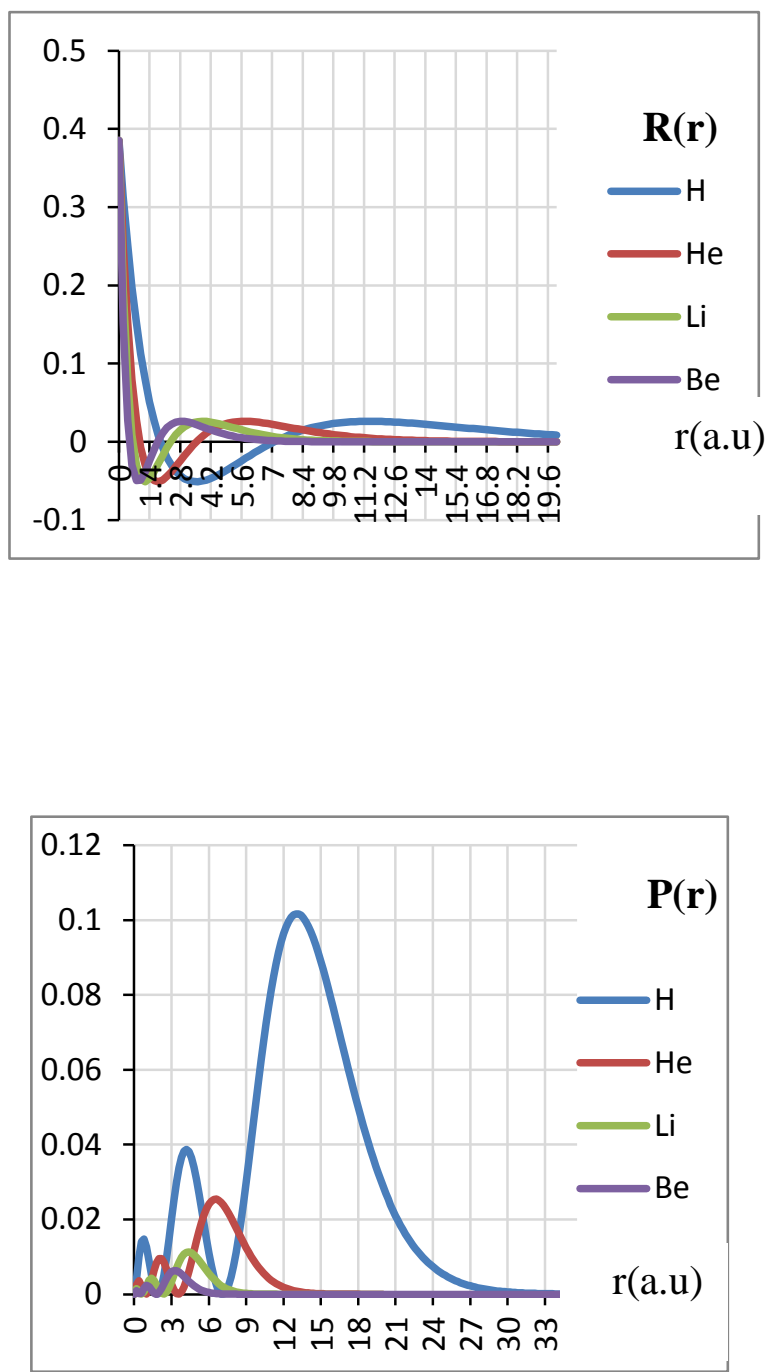

Fig. (4) The normalized radial wave function $R(r)$ and the radial distribution function $\mathrm{P}(\mathrm{r})$ for the $3 \mathrm{~s}$ atomic orbital in the hydrogen atom and hydrogen like ions. 


\begin{tabular}{|c|c|c|}
\hline $\mathbf{r}$ & $\mathbf{R}(\mathbf{r})$ & PH(r) \\
\hline 0 & 0 & 0 \\
\hline 0.2 & 0.021896684 & $1.91786 \mathrm{E}-05$ \\
\hline 0.4 & 0.039556278 & 0.000 \\
\hline 0.6 & 0.053525342 & 0.001031386 \\
\hline 0.8 & 0.064291684 & 0.002645389 \\
\hline 1 & 0.072290048 & 0.00 \\
\hline 1.2 & 0.077 & 0.00 \\
\hline 1.4 & 0.081487022 & 0.013014664 \\
\hline 1.6 & 0.08 & 0.01 \\
\hline 1.8 & & \\
\hline 2 & 0.08 & 0.02 \\
\hline 2.2 & 0.08 & 0.03 \\
\hline 2.4 & & \\
\hline 2.6 & 0.07 & 0.0 \\
\hline 2.8 & 0.07 & 0.03 \\
\hline 3 & 0.06 & \\
\hline 3.2 & 0.06 & 0.0 \\
\hline 3.4 & 0.05 & 0.03 \\
\hline 3.6 & 0.05 & \\
\hline 3.8 & 0.04 & \\
\hline 4 & 0.04 & 0.0 \\
\hline 4.2 & 0.037 & 0.02 \\
\hline 4.4 & 0.032 & \\
\hline 4.6 & 0.028 & 0.01 \\
\hline 4.8 & 0.023 & 0.012 \\
\hline 5 & & \\
\hline 5.2 & 0.01 & 0.0 \\
\hline 5.4 & 0.010 & 0.003405368 \\
\hline 5.6 & 0.006989375 & 0.001531979 \\
\hline 5.8 & 0.003386065 & 0.000385697 \\
\hline 6 & 0 & 0 \\
\hline 6.2 & & \\
\hline 6.4 & -0.00611813 & 0.001533193 \\
\hline 6.6 & -0.00885362 & 0.003414516 \\
\hline 6.8 & -0.01137814 & 0.00598633 \\
\hline 7 & -0.01369675 & 0.009192449 \\
\hline
\end{tabular}

\begin{tabular}{|c||c|c|c|c|}
\hline Atom & H & He & Li & Be \\
\hline$Z$ & 1 & 2 & 3 & 4 \\
\hline$\rho$ & $\mathbf{0 . 6 6 6 7}$ & 1.3333 & 2 & 2.6667 \\
\hline
\end{tabular}

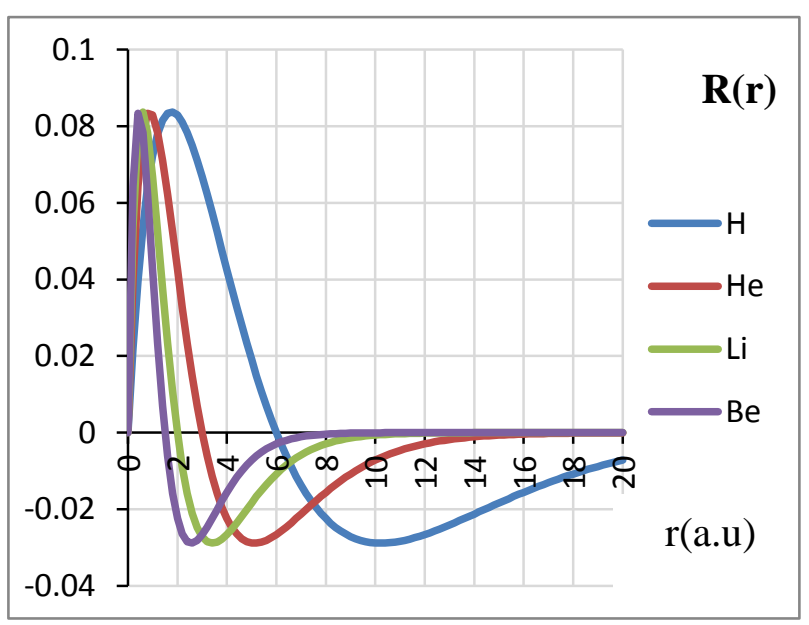

Fig. (5) The normalized radial wave function $R(r)$ and the radial distribution function $P(r)$ for the $3 p$ atomic orbital in the hydrogen atom and hydrogen like ions.

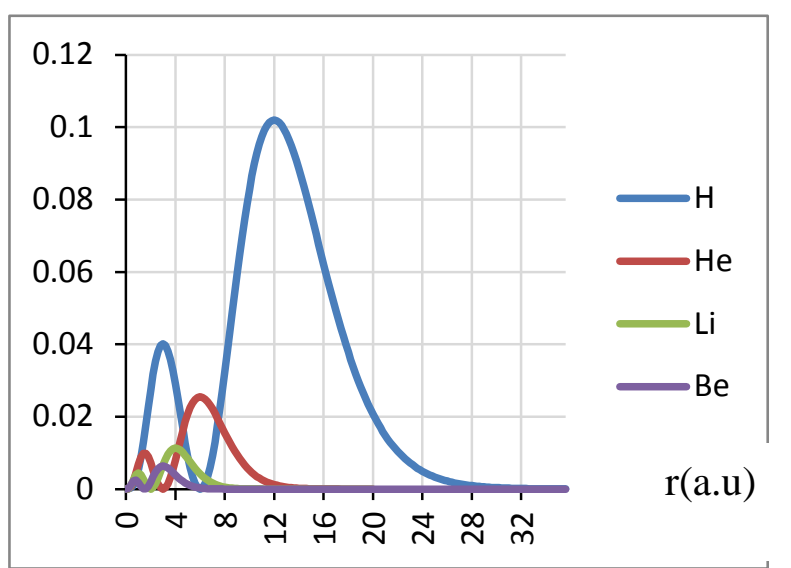

\begin{tabular}{|l|l|l|}
\hline $\mathbf{r}$ & $\mathbf{R H}(\mathbf{r})$ & $\mathbf{P H}(\mathbf{r})$ \\
\hline
\end{tabular}




\begin{tabular}{|c|c|c|}
0 & 0 & 0 \\
\hline 0.2 & 0.000337614 & $4.55933 \mathrm{E}-09$ \\
\hline 0.4 & 0.001263361 & $2.55373 \mathrm{E}-07$ \\
\hline 0.6 & 0.002659237 & $2.54576 \mathrm{E}-06$ \\
\hline 0.8 & 0.00442264 & $1.25182 \mathrm{E}-05$ \\
\hline 1 & 0.006464705 & $4.17924 \mathrm{E}-05$ \\
\hline 1.2 & 0.008708798 & 0.000109214 \\
\hline 1.4 & 0.011089165 & 0.00024102 \\
\hline 1.6 & 0.013549703 & 0.000470002 \\
\hline 1.8 & 0.016042862 & 0.00083389 \\
\hline 2 & 0.018528653 & 0.001373244 \\
\hline 2.2 & 0.020973758 & 0.002129109 \\
\hline 2.4 & 0.023350728 & 0.003140677 \\
\hline 2.6 & 0.025637263 & 0.00444314 \\
\hline 2.8 & 0.027815577 & 0.006065857 \\
\hline 3 & 0.029871811 & 0.008030926 \\
\hline 3.2 & 0.031795527 & 0.010352185 \\
\hline 3.4 & 0.033579246 & 0.01303466 \\
\hline 3.6 & 0.035218037 & 0.016074419 \\
\hline 3.8 & 0.036709153 & 0.019458795 \\
\hline 4 & 0.038051711 & 0.023166924 \\
\hline 4.2 & 0.0392464 & 0.027170538 \\
\hline 4.4 & 0.040295229 & 0.031434939 \\
\hline 4.6 & 0.041201306 & 0.035920107 \\
\hline 4.8 & 0.041968633 & 0.040581876 \\
\hline 5 & 0.042601942 & 0.045373136 \\
\hline 5.2 & 0.043106534 & 0.050245005 \\
\hline 5.4 & 0.043488154 & 0.055147961 \\
\hline 5.6 & 0.043752869 & 0.060032874 \\
\hline 5.8 & 0.043906974 & 0.064851945 \\
\hline 6 & 0.0439569 & 0.069559526 \\
\hline 6.2 & 0.043909144 & 0.074112817 \\
\hline 6.4 & 0.043770204 & 0.078472429 \\
\hline 6.6 & 0.043546528 & 0.082602831 \\
\hline 6.8 & 0.043244464 & 0.086472669 \\
\hline 7 & 0.04287023 & 0.090054972 \\
\hline & & \\
\hline
\end{tabular}

\begin{tabular}{|c|c|c|c|c|}
\hline Atom & $\mathbf{H}$ & $\mathbf{H e}$ & $\mathbf{L i}$ & $\mathbf{B e}$ \\
\hline $\mathbf{Z}$ & 1 & 2 & 3 & 4 \\
\hline $\mathbf{P}$ & 0.6667 & 1.3333 & 2 & 2.6667 \\
\hline
\end{tabular}
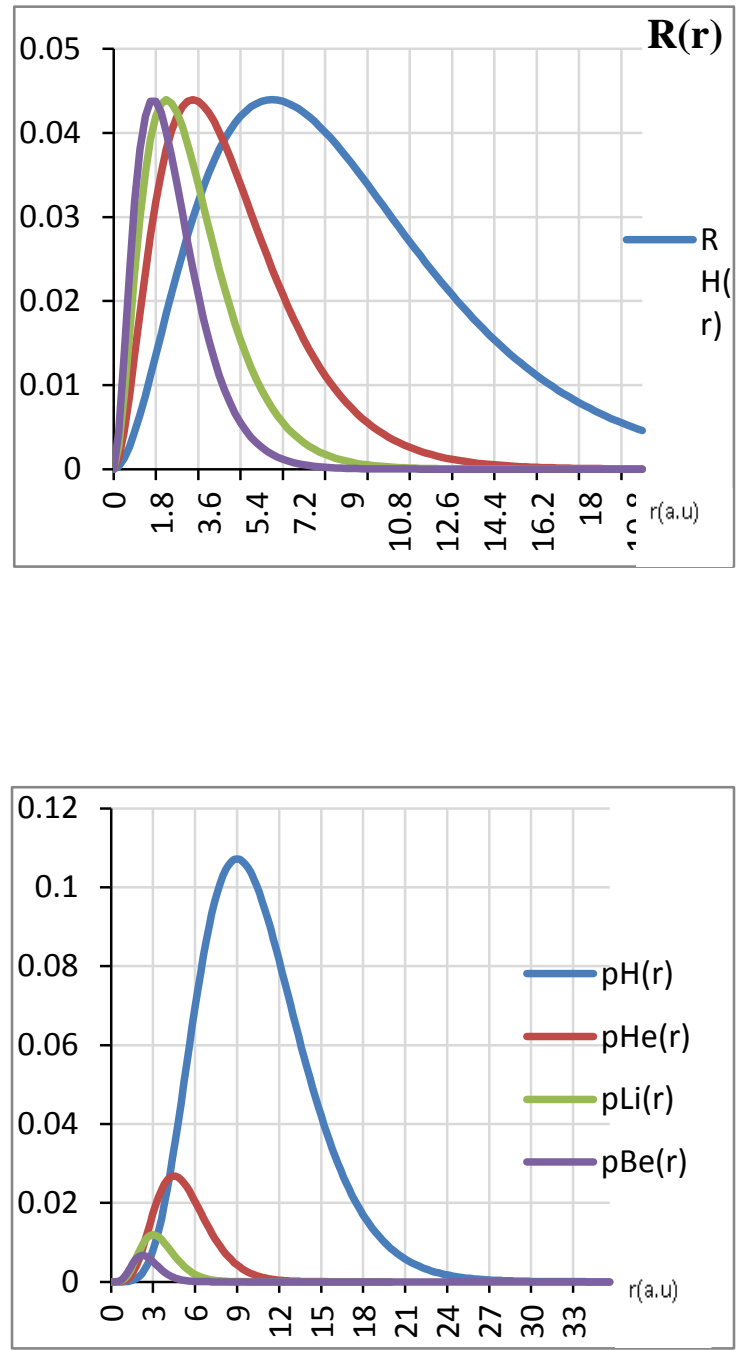

Fig. (6) The normalized radial wave function $R(r)$ and the radial distribution function $P(r)$ for the $3 d$ atomic orbital in the hydrogen atom and hydrogen like ions.

\section{Conclusion:}


function and probability density for the different orbits of the hydrogen atom and hydrogen like ions. From present work, we noted the following:

1- In $s$ orbits, the $R(r)$ value is not equal to zero at the nucleus, that can be observed in figures $(1,2,4)$, what not observed in other orbits.

2 - The probability of finding an electron in $2 s$ shell within one Bohr radius of the nucleus greater than the probability of finding the electron in $2 p$ shell in the same area, this shows that the electrons $1 s$ more effective in blocking electrons of $2 p$ from the nuclear shipment than in the blocking electrons of $2 s$. in other words, the orbits $2 s, 2 p$ will have the same energy in the atoms that have more than one electron.

3 - Figures (1..6), show that the distribution function of the hydrogen atom is greater than the distribution function of ions that hydrogen like ions. Whenever an atomic number increasing, the function will approach to the nucleus, according to the force of attraction between the nucleus and the electron.

\section{References:}

1- Adel,H.Ban,Al-bayati,K.H.2011. calculate the one -expectation to electronic charge of atomic system continue two electron, Baghdad Sci. J. 7(1):6-9.

2- Steiner , E. 1996 . the chemistry maths Book , Oxford Science publications, Oxford.

3- March , N.H. , 2003. Local energy equation for two- electron atoms and relation between kinetic energy and electron densities ,J. chem. phys 118(15):6846-6851.

4- Charles, M . 2008 . Quinn, computational chemistry An interactive Guide to Basis Set Theory , The National University of Ireland, Ireland.

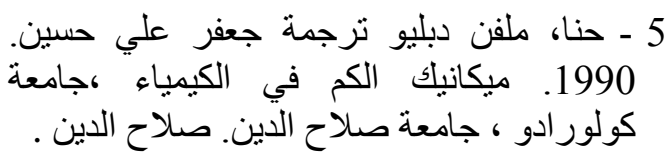

$$
\begin{aligned}
& \text { حساب كثافة الاحتمالية القطرية والدالة القطرية لذرة الهيدروجين والايونات }
\end{aligned}
$$

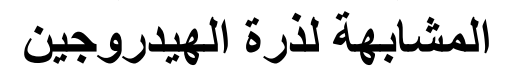

$$
\begin{aligned}
& \text { *سرين خليل عبل الامبير }
\end{aligned}
$$

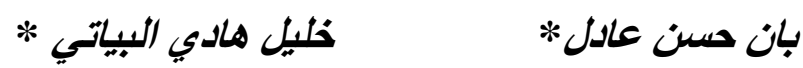

$$
\begin{aligned}
& \text { *جامعة بغداد / كلبة العلوم للبنات / قسم الفيزياء } \\
& \text { الخلاصة : مان }
\end{aligned}
$$

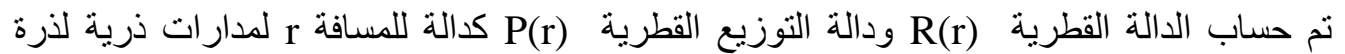

$$
\begin{aligned}
& \text { الهيدروجين و قورنت النتائج مع الايونات المشابهة لذرة الهيدروجين ( }
\end{aligned}
$$

